\title{
Evaluation of cases with a preliminary diagnosis of Crimean- Congo hemorrhagic fever and comparison of characteristics in patients admitted to a secondary care hospital in Kastamonu, Turkey.
}

\author{
Hüseyin Can Hekimoğlu, Neşe Ateş Demirci
}

Kastamonu Dr. Münif Islamoğlu Hospital, Infectious Diseases and Clinical Microbiology Department

\begin{abstract}
:
Background: Crimean-Congo hemorrhagic fever (CCHF) is an endemic disease in Turkey. The clinical presentation and laboratory findings are not specific especially in cases without hemorrhagic findings.

Objective: We aimed to evaluate CCHF cases and compare them with non-CCHF cases in terms of their characteristics during admission.

Methods: Cases with a preliminary diagnosis of CCHF at a secondary care hospital in Kastamonu in 2013 were evaluated, retrospectively. Cases testing RNA/IgM positive were considered as CCHF. Cases testing both RNA and IgM negative were considered as non-CCHF. The two groups were then compared in terms of their clinical, laboratory and epidemiological characteristics during admission.

Results: A total of 41 cases were tested and CCHF was found in $46.3 \%$ of cases. Fatality was $5.3 \%$ in CCHF cases. The frequency of tick bites and CK elevation in CCHF cases was significantly higher than non-CCHF cases $(\mathrm{p}<0.05)$. There were no significant differences between the two groups regarding other characteristics ( $\mathrm{p}>0.05)$.

Conclusions: In cases with a preliminary diagnosis of CCHF, especially in cases without a history of tick bite and with normal CK levels during admission, performing tests for the differential diagnosis may be advisable without waiting for the results of tests for CCHF.
\end{abstract}

Kewords: Crimean-congo hemorrhagic fever, Kastamonu, Turkey

DOI: http://dx.doi.org/10.4314/ahs.v14i4.15

\section{Introduction}

Crimean-Congo hemorrhagic fever (CCHF) is a tickborne viral zoonotic infection acquired by a tick bite, transmitted from body fluids or blood of domestic animals and cases with CCHF virus (CCHFV); this virus belongs to the genus Nairovirus in the family Bunyaviridae. The clinical spectrum of the disease varies from a subclinical infection to severe disease and death, with a reported fatality rate of $15-30 \%$ in human cases ${ }^{1-10}$. It was reported in the Crimean Peninsula in 1944 for the first time ${ }^{11}$. Since then, CCHF has been reported from different parts of Africa, Eastern Europe, the Balkans, the Middle East, Central and Southern Asia', $10,12,13$. The first case of CCHF infection in Turkey was reported in the Kelkit Valley region in 2002.

\section{Corresponding author: \\ Hüseyin Can Hekimoğlu \\ Kastamonu Dr. Münif Islamoğlu Hospital, \\ Infectious Diseases and Clinical Microbiology \\ Department \\ drchh@hotmail.com}

Turkey has become the country with the highest number of $\mathrm{CCHF}$ cases among the countries that report CCHF cases annually. This infection is an endemic zoonosis appearing every year during spring and summer in Turkey with approximately 1000 cases reported annually ${ }^{1-10}$. Majority of cases in Turkey were from 15 cities in Kelkit Valley and its environs including Kastamonu Province. CCHF cases from countries that border Turkey including Bulgaria, Greece, Iran and Iraq have also been reported ${ }^{6,13-18}$. According to various studies, the fatality rate of CCHF ranged from 15-30\%. However, the crude fatality rate was 5\% in 2002-2007 according to the reports of the Turkish Ministry of Health surveillance $^{1-10}$.

There are a few studies evaluating cases with a preliminary diagnosis of $\mathrm{CCHF}$ and reporting the rate of confirmed CCHF cases among suspected CCHF cases. This rate ranges between $21 \%$ and $57 \%{ }^{19-22}$. In this study, cases of CCHF admitted to a secondary care hospital in Kastamonu in 2013 were evaluated in terms of their clinical, laboratory and epidemiological characteristics. Cases with CCHF were also compared in terms of these characteristics with non-CCHF cases whose reverse transcriptase-polymerase chain 
reaction (RT-PCR) and ELISA IgM tests were negative for CCHFV.

\section{Methods}

In 2013, cases admitted with a preliminary diagnosis of CCHF to the Dr Münif Islamoğlu Hospital (Kastamonu Province, Turkey) were included in this study. A preliminary diagnosis of CCHF was made if cases presented with at least two of: (a) sudden onset of highgrade fever; (b) headache; (c) weakness; (d) nausea and vomiting; (e) diarrhea; and one of: (a) thrombocytopenia (platelet count of $<150 \times 10^{9} / \mathrm{L}$ ); and/or (b) leucopenia (white blood cell count of $<4 \times 10^{9} / \mathrm{L}$ ); and also one of: (a) history of tick bite; (b) close contact with animals; (c) living in rural areas or travelling to rural areas; (d) being a laboratory worker; or (e) contact with people with similar symptoms in the past 15 days.

Serum samples of all cases were collected within 24 hours of admission and were sent to the Public Health Institution of Turkey, National Virology Reference Laboratory to determine CCHF IgM antibodies by ELISA and CCHFV RNA by RT-PCR. ELISA was not performed on serum samples already detected by RTPCR as positive for viral RNA. Cases with viral RNA or IgM antibody positivite were considered as CCHF cases. Cases with both viral RNA and IgM antibody negative were considered as non-CCHF cases. Cases with a history of suspected CCHF in the past four months were excluded from the study because, serum CCHF IgM antibodies detected by ELISA remain positive for four months after the infection ${ }^{2,4,9,10}$. The incubation period of cases with tick exposure was defined as the period between contact with tick and onset of symptoms. Pediatric cases ( $\leq 16$ ages) were excluded from the study.

Moreover, there were no pediatric cases with a definite diagnosis of CCHF in our hospital in 2013. CCHF cases were evaluated retrospectively in terms of their clinical and laboratory features during admission to the hospital and epidemiological features within 15 days, and these characteristics of CCHF cases were compared with non-CCHF cases.

Statistical analysis was performed using SPSS version 15-0 (SPSS Inc., USA). Descriptive statistics (median, minimum and maximum value, mean and standard deviation, count and percentage) were used to summarize the results. Nominal variables were compared using $\chi^{2}$ test with Yates' correction and Fisher's exact test. Continuous variables were compared using Mann Whit ney $\mathrm{U}$ test. When a p-value was found less than 0.05 , the result was considered as statistically significant and the null hypothesis was rejected.

\section{Result}

In 2013, a total of 41 cases with a preliminary diagnosis of CCHF were followed up at our hospital. Three had positive IgM by ELISA and 16 cases had a positive RTPCR for CCHFV RNA. A total of 19 cases $(46.3 \%)$ were diagnosed with definite CCHF. The remaining 22 cases $(53.7 \%)$ were found negative by both tests and were considered as non-CCHF cases. $52.6 \%$ (10) of the CCHF cases were female, while $47.4 \%$ (9) were male. Besides, $54.5 \%$ (12) and $45.5 \%$ (10) of the non-CCHF cases were female and male, respectively. The median age of CCHF cases and non-CCHF cases was 54.0 (16$83)$ and 51.5 (20-78), respectively. There were no statistically significant differences between the two group regarding gender and age ( $p>0.05$ ). Six cases from the $\mathrm{CCHF}$ group were transferred to a tertiary care hospital. One of the transferred CCHF cases died while the other 18 cases were cured. Case who died was a 42 year old female patient with no comorbid diseases and the cause of her death was multi organ failure. She did not receive ribavirin therapy. Thus, fatality rate was $5.3 \%$ $(1 / 19)$ in the CCHF group. Three of the transferred cases in the CCHF group and none of non-CCHF cases recieved oral ribavirin.

When looking at the monthly distribution of the $\mathrm{CCHF}$ cases, it was shown that the first case was detected in April and the highest number of cases was admitted in May (Figure 1)

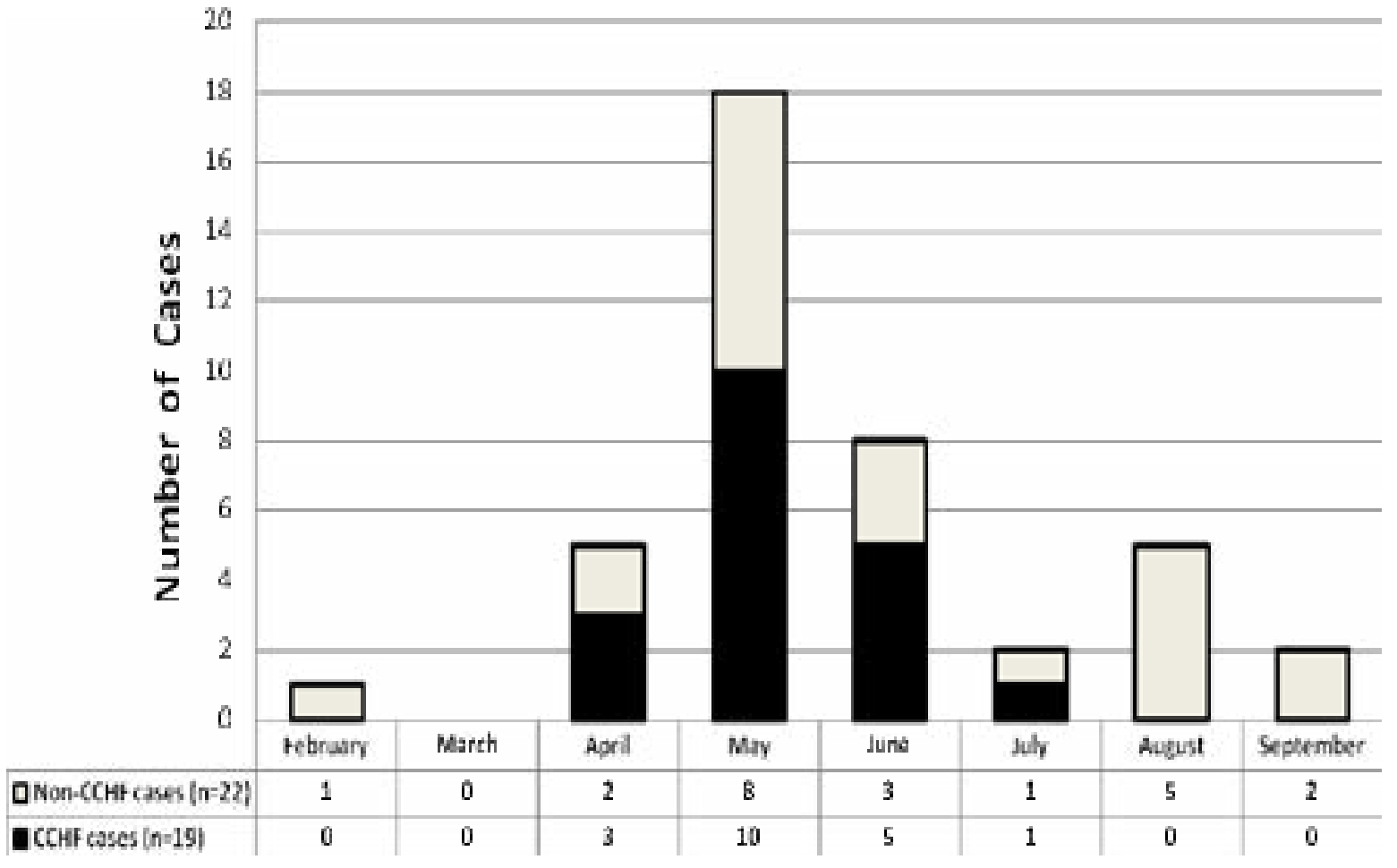

Five cases were from Daday and four cases were from tamonu. The distribution of cases with a preliminar Devrekani and Taskopru which are districts of Kas- diagnosis of CCHF by districts is shown in Figure 2 .

Figure 2: The distribution of cases with a preliminary diagnosis of Crimean- Congo hemorrhagic fever by districts.

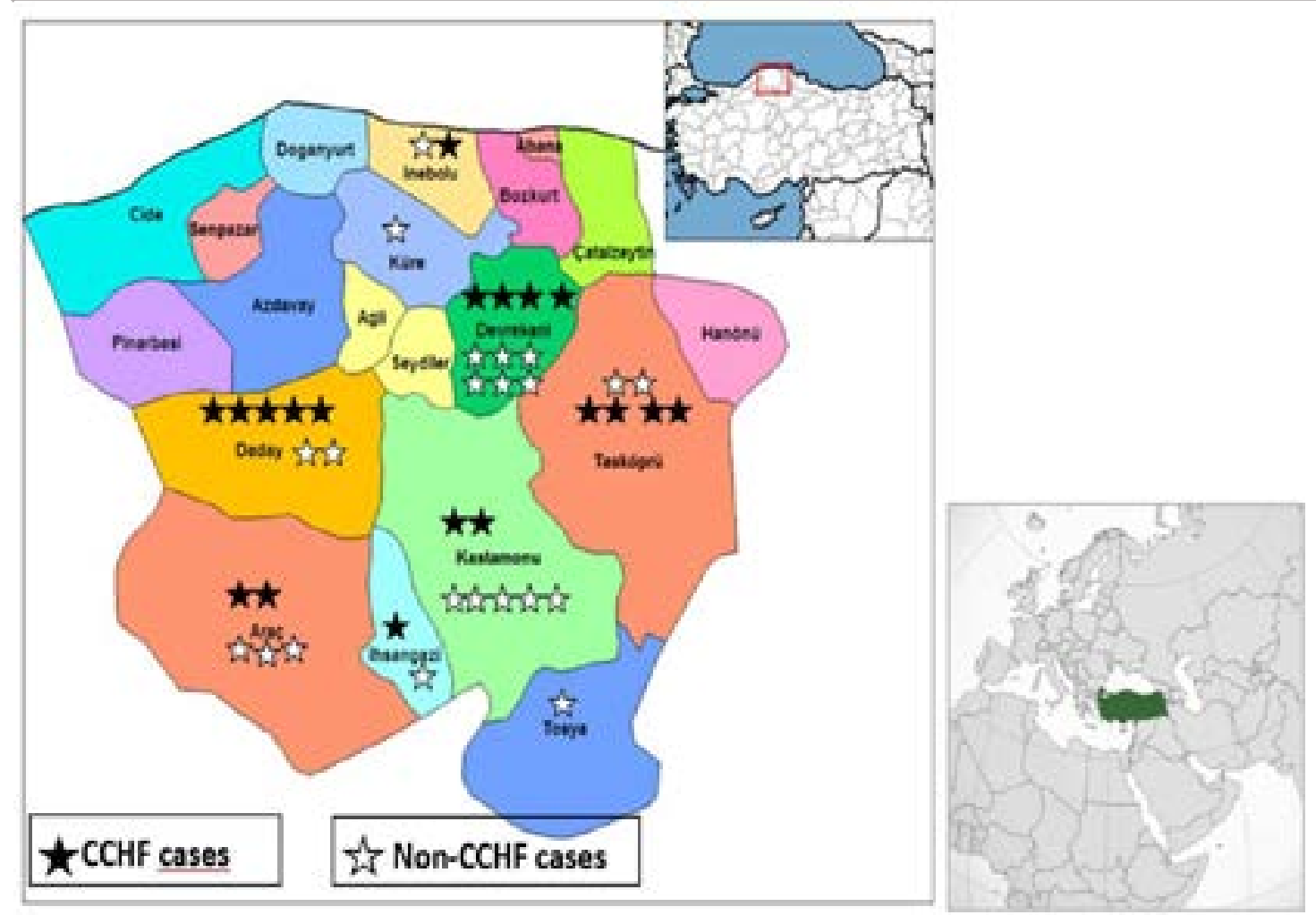


In both groups, the most common clinical symp- Bleeding was observed in four $(18.2 \%)$ cases in the toms during admission were weakness, widespread non- CCHF group during admission. There was no muscle pain, fever, headache, nausea and vomiting, re- bleeding during admission in the CCHF group. There spectively. The frequency of abdominal pain, diarrhea were no statistically significant differences between the and maculopapular rash was higher in CCHF cases. two groups in terms of clinical symptoms during admission ( $\mathrm{p}>0.05)$ (Table 1)

Table 1: Clinical symptoms of cases with a preliminary diagnosis of

Crimean-Congo hemorrhagic fever during admission.

\begin{tabular}{lccc}
\hline & $\begin{array}{c}\text { CCHF } \\
\text { cases (19) }\end{array}$ & $\begin{array}{c}\text { Non-CCHF } \\
\text { cases (22) }\end{array}$ & p value \\
\hline Clinical symptoms & $\%$ (n) & $\%(n)$ & \\
Weakness & & & \\
Widespread muscle pain & $100(19)$ & $95.5(21)$ & $\mathrm{p}>0.05$ \\
Fever & $94.7(18)$ & $86.4(19)$ & $\mathrm{p}>0.05$ \\
Headache & $84.2(16)$ & $72.7(16)$ & $\mathrm{p}>0.05$ \\
Nausea and vomiting & $84.2(16)$ & $72.7(16)$ & $\mathrm{p}>0.05$ \\
Abdominal pain & $84.2(16)$ & $68.2(15)$ & $\mathrm{p}>0.05$ \\
Diarrhea & $36.8(7)$ & $31.8(7)$ & $\mathrm{p}>0.05$ \\
Maculopapular rash & $36.8(7)$ & $18.2(4)$ & $\mathrm{p}>0.05$ \\
Altered level of consciousness & $26.3(5)$ & $18.2(4)$ & $\mathrm{p}>0.05$ \\
Bleeding* & $10.5(2)$ & $4.5(1)$ & $\mathrm{p}>0.05$ \\
& $0.0(0)$ & $18.2(4)$ & $\mathrm{p}>0.05$
\end{tabular}

* Includes hematoma,hemoptysis,hematuria and nose, gingival, vaginal, gastrointestinal,

intra-abdominal and intracranial bleeding.

The most common physical finding during admis- between the two groups in terms of physical findsion was fever, which was present in $68.4 \%$ (13) of ings during admission ( $p>0.05$ ). The most common CCHF and $50 \%$ (11) of non-CCHF cases. In the CCHF laboratory findings in the cases with a preliminary group, five $(26.3 \%)$ cases had maculopapular rash, diagnosis of CCHF were thrombocytopenia, leukopetwo $(10.5 \%)$ had altered level of conscious- nia, and aspartate aminotransferase (AST) and alanine ness and hypotension, and one (5.3\%) had ecchymo- aminotransferase (ALT) elevation, respectively. $94.7 \%$ sis, petechiae and tachycardia during admission. In the (18) of CCHF group and 95.5\% (21) of non-CCHF non-CCHF group, four (18.2\%) cases had hemorrhagic group had thrombocytopenia. The proportion of cases findings, three $(13.6 \%)$ had tachycardia, two $(9.1 \%)$ with platelet counts between $150-101 \times 10^{9} / \mathrm{L}, 100-51$ had ecchymosis and hypotension, and one (4.5\%) $\times 10^{9} / \mathrm{L}$ and $<51 \times 10^{9} / \mathrm{L}$ was $26.3 \%(5), 52.6 \%(10)$ had maculopapular rash and altered level of conscious- $15.8 \%$ (3) in the CCHF group and $54.5 \%(12), 27.3 \%$ ness. There was no statistically significant difference (6) $13.6 \%$ (3) in the non-CCHF group, respectively.
While elevated creatine phosphokinase (CK) and frequency of CK elevation in CCHF cases (57.9\%) durlactate dehydrogenase (LDH) levels were detected ing admission was significantly higher than non- CCHF more frequently in the CCHF group, anemia was cases $(22.7 \%)(\mathrm{p}<0.05)$. Other laboratory findings were detected more frequently in the non-CCHF group. The similar between the two groups ( $p>0.05)$ (Table 2).

Table 2: The distribution of the laboratory findings of cases with a

preliminary diagnosis of Crimean-Congo hemorrhagic fever during admission.

\begin{tabular}{|c|c|c|c|}
\hline & $\begin{array}{c}\text { CCHF } \\
\text { cases (19) }\end{array}$ & $\begin{array}{l}\text { Non-CCHF } \\
\text { cases }(22)\end{array}$ & p value \\
\hline Laboratory findings & $\%(n)$ & $\%(n)$ & \\
\hline Thrombocytopenia & $94.7(18)$ & $95.5(21)$ & $\mathrm{p}>0.05$ \\
\hline \multicolumn{4}{|c|}{ Platelet count ranges from: } \\
\hline $150-101 \times 10^{9} / \mathrm{L}$ & $26.3(5)$ & $54.5(12)$ & $\mathrm{p}>0.05$ \\
\hline $100-51 \times 10^{9} / \mathrm{L}$ & $52.6(10)$ & $27.3(6)$ & $\mathrm{p}>0.05$ \\
\hline $50.000-0 \times 10^{9} / \mathrm{L}$ & $15.8(3)$ & $13.6(3)$ & $\mathrm{p}>0.05$ \\
\hline Leukopenia & $89.5(17)$ & $63.6(14)$ & $\mathrm{p}>0.05$ \\
\hline AST-ALT elevation & $84.2(16)$ & $59.1(13)$ & $\mathrm{p}>0.05$ \\
\hline LDH elevation & $68.4(13)$ & $59.1(13)$ & $\mathrm{p}>0.05$ \\
\hline CK elevation & $57.9(11)$ & $22.7(5)$ & $P<0.05$ \\
\hline Anemia & $47.4(9)$ & $63.6(14)$ & $\mathrm{p}>0.05$ \\
\hline INR elevation & $38.8(7)$ & $22.7(5)$ & $\mathrm{p}>0.05$ \\
\hline
\end{tabular}

When the cases were evaluated according to their within 15 days before the onset of symptoms. In nonoccupations, majority were farming-animal husbandry $\mathrm{CCHF}$ cases, these proportions were $86.4 \%(19), 77.3 \%$ with $68.2 \%(15)$ and $73.7 \%(14)$ in the CCHF and the $(17)$ and $27.3 \%(6)$, respectively. In CCHF cases, a his-
non- CCHF groups, respectively. The remaining cases tory of tick bite in the last 15 days was significantly had no occupational risk. Among CCHF cases, 94.7\% higher than non-CCHF cases $(p<0.05)$. Statisti(18) were living in rural areas, $89.5 \%$ (17) had contact cally significant differences were not found between with animals and $84.2 \%$ (16) had a history of tick bite the two groups in terms of other epidemiological characteristics $(\mathrm{p}>0.05)$ (Table 3$)$. 
Table 3. The distribution of epidemiological characteristics of cases with

a preliminary diagnosis of Crimean-Congo hemorrhagic fever within the last 15 days.

\begin{tabular}{lccc}
\hline & $\begin{array}{c}\text { CCHF cases } \\
\mathbf{( 1 9 )}\end{array}$ & $\begin{array}{c}\text { Non-CCHF } \\
\text { cases (22) }\end{array}$ & p value \\
\hline Epidemiological characteristics & $\mathbf{\% ( n )}$ & $\mathbf{\%}(\mathbf{n})$ & \\
& & & \\
Living in rural areas & $94.7(18)$ & $86.4(19)$ & $\mathrm{p}>0.05$ \\
Contact with animals & $89.5(17)$ & $77.3(17)$ & $\mathrm{p}>0.05$ \\
Tick bite & $84.2(16)$ & $27.3(6)$ & $\mathbf{P}<0.05$ \\
Contact with body fluids, tissue & & & \\
or blood of animals & $42.1(8)$ & $36.4(8)$ & $\mathrm{p}>0.05$ \\
Traveling to rural areas & $10.5(2)$ & $18.2(4)$ & $\mathrm{p}>0.05$ \\
Contact with CCHF cases & $0.0(0)$ & $0.0(0)$ & $\mathrm{p}>0.05$ \\
\hline
\end{tabular}

The mean incubation period of cases with tick exposure with a preliminary diagnosis of CCHF and there was no was $3.8 \pm 3.3$ days in the CCHF group. The median significant difference between the two groups in terms day of illness on which patients were admitted was 2.0 of gender $(\mathrm{p}>0.05)$.

$(0-6)$ and $1.5(0-9)$ days in CCHF and

patients, respectively and the difference was not sta- The fatality rate of CCHF was approximately $15-30 \%$ tistically significant $(p>0.05)$. The median elapsed time $1-10$. The mean fatality rate for Turkey was about $5 \%$ from admission of cases to the conclusion of RT-PCR in 2002-2007. The fatality rate was calculated as $8.8 \%$ and/or ELISA was 3.0 (1-6) days for CCHF cases, while in Bolu Province located in the Western Black Sea reit was $5.0 \pm(2-10)$ days for non-CCHF cases. In the gion of Turkey in 2006-2012 ${ }^{21}$. In our hospital, 342 non-CCHF group, the elapsed time was significantly CCHF cases were diagnosed in 2005-2010 and the fatalhigher than the CCHF group $(\mathrm{p}<0.05)$. ity rate was found to be $2.9 \% \%^{29}$. In 2013 , we diagnosed 19 CCHF cases and the fatality rate was $5.3 \%$. Although this rate was similar to that of other parts of Turkey,

\section{Discussion}

The number of male and female CCHF cases in Turkey is similar. Two thirds of cases are farmers or housewives. Housewives in rural areas work actively particularly in farming and animal husbandry in Turkey ${ }^{23-27}$. No housewives were included in our study, because, housewives live mostly in rural areas and work in animal husbandry and farming sector. Between 2004-2007, the proportion of health care workers among CCHF cases was $0.4 \%$ in Turkey and seroprevalence among healthcare workers in endemic areas was $2 \%{ }^{6,28}$. In our study, there was no health care worker. In Iran, $34.3 \%$ of the 2536 cases with suspicion of CCHF was confirmed as CCHF and the probability of positive results for male samples was higher than that for female samples during 1999-2012 ${ }^{20}$. In our study, the proportion of CCHF cases was $46.3 \%$ among case incidence was in May and there were no cases detected in August. However, besides our hospital, there is one more private hospital that could follow up cases with CCHF in Kastamonu. Therefore, CCHF cases might have been admitted to this hospital, or a hospital outside of Kastamonu, during the same period.

The most common symptoms in cases with CCHF are fever, headache, myalgia, nausea, vomiting, abdominal pain, diarrhea and hemorragic findings, respectively $y^{6,9,10}$. In some studies, fever, bleeding, vomiting, headache and diarrhea were reported more frequently in cases with CCHF than non-CCHF cases ${ }^{20,21}$. In our study, there were no significant differences between the two groups in terms of clinical findings during admission $(p>0.05)$. Unexpectedly, there were no hemorrhagic findings among CCHF cases during admission. This may be due to the fact that patients were in early phase of CCHF. Because, in our study, the median day of illness on which CCHF cases were admitted was 2.0 days. On the other hand, this may be due to the fact that cases with hemorrhagic findings were admitted to other clinics/hospitals and/or they were misdiagnosed in other departments.

Thrombocytopenia, leucopenia, increased AST-ALT, $\mathrm{CK}$ and LDH levels are the most common laboratory findings in CCHF cases ${ }^{6,9},{ }^{10}$. In some studies, thrombocytopenia, increased AST-ALT and LDH levels were reported more commonly among cases with CCHF than non-CCHF cases ${ }^{20,21}$. In our study, CK elevation was more common in CCHF cases $(\mathrm{p}<0.05)$, but, other laboratory findings during admission were found to be similar between the two groups $(\mathrm{p}>0.05)$. Increased CK levels during admission in cases with CCHF may help to distinguish similar cases but our CCHF cases had milder clinical signs. This may account for the results of other laboratory investigation that were not significantly different.

A specific diagnosis may be made by testing a serum specimen for viral RNA by RT- PCR and for virus-specific IgM and/or IgG by ELISA or other methods. In general, virus can be detected for up to two weeks after the first clinical symptoms occur. An IgM antibody response is detectable from the fourth day after the onset of disease for up to four months. IgG antibodies can be found from sixth day after the incubation period up to five years $2,4,9,10$. In our study, the mean elapsed time from the admission of cases to the conclusion of tests was higher for non-CCHF cases than CCHF cases. As our hospital is a secondary care hospital having limited diagnostic facilities, excluding the diagnosis of CCHF can take more time. Therefore, performing tests for the differential diagnosis may be advisable during this time.

CCHFV transmission may occur by the bite of an infected tick or by exposure to the body fluids of a viremic animal or a CCHF case ${ }^{2,4,9,10}$. Tick bites and animal husbandry have been reported as risk factors for seropositivity and a history of tick bite and animal husbandry was identified in majority of CCHF cases in Turkey. Seroprevalence was 10\% in individuals with a history of tick bite. Seroprevalence was reported as $10-19.6 \%$ in endemic regions of Turkey. Seropositivity in individuals with a history of farming, animal husbandry, contact with animals and contact with ticks was $80 \%, 70 \%, 76 \%$ and $70 \%$ respectively $y^{6,8,30,31-34}$. In Iran contact with infected humans and animals was higher in the CCHF group than the non-CCHF group, but, a history of tick bite was not a risk factor ${ }^{20}$. Similarly, contact with animals was more commonly reported in CCHF cases than non-CCHF cases, but, a history of tick bite was similar between the two groups in Bolu, Turkey ${ }^{21}$. We found that the frequency of tick bites was significantly higher in CCHF cases than non-CCHF cases $(\mathrm{p}<0.05)$.

The efficacy of ribavirin in antiviral treatment remain controversial. Although most reports claimed a therapeutic benefit about ribavirin use, the quality of the evidence was low. In general, treatment of CCHF is mainly supportive therapy ${ }^{9,10,35,36}$. In our study, the case who died did not receive ribarivin therapy and three of CCHF cases received oral ribavirin.

This study has several limitations. First, the study reports a retrospective, one-year, single center data and includes only participants who were admitted to our hospital. Second limitation is small sample size and low study power. Significant differences between the two groups may have not been detected. Third, the study evaluates characteristics of cases during admission, the patients were not evaluated during the course of the disease. Despite these limitations, all patients admitted to our hospital were included the study. There are a few studies evaluating cases with a preliminary diagnosis of CCHF ${ }^{19-22}$. In this regard, there is a need for more comprehensive research. 
The disease still remains important in endemic regions rhagic fever: history, epidemiology, pathogenesis, clini-

of Turkey, although the fatality rate is low (5\%) in Tur- cal syndrome and genetic diversity. Antiviral Res 2013; cal presentation is not diagnostic and physical examination and laboratory findings are not specific especially in cases without hemorrhagic findings $\mathrm{s}^{2-4,8-10}$. In areas endemic for CCHF, clinicians should be aware of the possibility of other diseases in cases with a preliminar diagnosis of CCHF and should determine and consider clinical, laboratory and epidemiological characteristics during admission of such cases.

\section{Conclusion}

In cases with a preliminary diagnosis of CCHF, especially in cases without a history of tick bite and with normal CK levels during admission, performing tests for the differential diagnosis may be advisable withou waiting for the results of RT-PCR and ELISA tests for CCHF. This may help reduce the number of cases misdiagnosed as CCHF and increase the overall probability of detecting CCHF cases.

\section{References}

1. Swanepoel R, Gill DE, Shepherd AJ, Leman PA, Mynhardt JH, Harvey S. The clinical pathology of Crimean-Congo hemorrhagic fever. Rev Infect Dis 1989; 11:794-800.

2. Whitehouse CA. Crimean-Congo haemorrhagic fever. Antiviral Res 2004; 64: 145-60.

3. Jamil B, Hasan RS, Sarwari AR, Burton J, Hewson R, Clegg C. Crimean-Congo hemorrhagic fever: experience at a tertiary care hospital in Karachi, Pakistan. Trans R Soc Trop Med Hyg 2005; 99: 577-84.

4. Ergönül O. Crimean-Congo haemorrhagic fever. Lancet Infect Dis 2006; 6: 203-14.

5. Chinikar S, Goya MM, Shirzadi MR, et al. Surveillance and laboratory detection system of CrimeanCongo haemorrhagic fever in Iran. Transbound Emerg Dis 2008; 55: 200-4.

6. Yilmaz GR, Buzgan T, Irmak H, et al. The epidemiology of Crimean-Congo hemorrhagic fever in Turkey, 2002-2007. Int J Infect Dis 2009; 13: 380-6.

7. Saijo M, Morikawa S, Kurane I. Recent progress in the treatment for Crimean- Congo haemorrhagic feve and future perspectives. Future Virol 2010; 5: 801-9.

8. Bodur H, Akinci E, Ascioglu S, Öngürü P, Uyar Y. Subclinical infections with Crimean-Congo hemorrhagic fever virus, Turkey. Emerg Infect Dis 2012; 18: 640-2.

9. Bente DA, Forrester NL, Watts DM, McAuley AJ, Whitehouse CA, Bray M. Crimean-Congo hemor100: 159-89.

10. Mertens M, Schmidt K, Ozkul A, Groschup MH The impact of Crimean-Congo hemorrhagic fever virus on public health. Antiviral Res 2013; 98: 248-60.

11. Hoogstraal H. The epidemiology of tick-borne Crimean-Congo hemorrhagic fever in Asia, Europe, and Africa. J Med Entomol 1979; 15: 307-417.

12. Sidira P, Maltezou HC, Haidich AB, Papa A Seroepidemiological study of Crimean-Congo haemorrhagic fever in Greece, 2009-2010. Clin Microbio Infect 2012; 18: E16-9.

13. Vorou RM, Papavassiliou VG, Tsiodras S. Emerging zoonoses and vector-borne infections affecting humans in Europe. Epidemiol Infect 2007; 135: 1231-47.

14. Karti SS, Odabasi Z, Korten V, et al. CrimeanCongo hemorrhagic fever in Turkey. Emerg Infect Dis 2004; 10: 1379-84.

15. Günaydın NS, Aydın K, Yılmaz G, Çaylan R, Köksal İ. Crimean-Congo hemorrhagic fever cases in the eastern Black Sea Region of Turkey: demographic geographic, climatic, and clinical characteristics. Turk J Med Sci 2010; 40: 829-34.

16. Chinikar S, Ghiasi SM, Hewson R, Moradi M, Haeri A. Crimean-Congo hemorrhagic fever in Iran and neighboring countries. J Clin Virol 2010; 47: 110-4.

17. Gergova I, Kunchev M, Kamarinchev B. CrimeanCongo haemorrhagic fever virustick survey in endemic areas in Bulgaria. J Med Virol 2012; 84: 608-14.

18. Ergonul O. Crimean-Congo haemorrhagic fever virus: new outbreaks, new discoveries. Curr Opin Viro 2012; 2: 215-20.

19. Erenler AK, Kulaksiz F, Ülger H, et al. Characteristics of patients admitted to the emergency department due to tick bite. Trop Doct 2014; 44: 86-8.

20. Mostafavi E, Pourhossein B, Chinikar S. Clinical symptoms and laboratory findings supporting early diagnosis of Crimean-Congo hemorrhagic fever in Iran. Med Virol 2014; 86: 1188-92.

21. Duran A, Küçükbayrak A, Ocak T, et al. Evaluation of patients with Crimean- Congo hemorrhagic fever in Bolu, Turkey. Afr Health Sci 2013; 13: 233-42.

22. Kuchuloria T, Imnadze P, Chokheli M, et al. Viral Hemorrhagic Fever Cases in the Country of Georgia Acute Febrile Illness Surveillance Study Results. Am J Trop Med Hyg 2014. pii: 13-0460

23. Izadi S, Holakouie-Naieni K, Majdzadeh SR, et al. Seroprevalence of Crimean- Congo hemorrhagic fever in Sistan-va-Baluchestan Province of Iran. Jpn J Infect Dis 2006; 59: 326-8.
24. Swanepoel R, Shepherd AJ, Leman PA, Shepherd J Med Virol 2014; 86: 306-14.

SP, Miller GB. A common- source outbreak of Crime- 31. Gunes T, Enoin A, Poyraz O, et al. Crimean-Congo Med J 1985; 68: 635-7. hemorrhagic fever virus in high-risk population, Tur-
key. Emerg Infect Dis 2009;15: 461-4.

32. Gargili A, Midilli K, Ergonul O, et al. CrimeCrimean-Congo haemorrhagic fever outbreak in Cen- an-Congo hemorrhagic fever in European part of tral Anatolia: a case control study. R2227. 15 th Euro- Turkey: Genetic analysis of the virus strains from ticks pean Congress of Clinical Microbiology and Infectious and a seroepidemiological study in humans. Vector Born Diseases (ECCMID). 2005

26. Vorou R, Pierroutsakos IN, Maltezou HC. Crime- 33. Ertugrul B, Kirdar S, Ersoy OS, et al. The seroprevan-Congo hemorrhagic fever.Curr Opin Infect Dis 2007; alence of Crimean-Congo haemorrhagic fever among 20: 495-500.

27. Ozkurt Z, Kiki I, Erol S. Crimean-Congo hemorrhagic fever in Eastern Turkey: clinical features, risk factors and efficacy of ribavirin therapy. J Infect 2006; 52: 207-15.

, H. The seroprevalance of Crimean-Congo hace rhagic fever in people living in the same environmen S, Barut S, Bursali A, et al. Sero- with Crimean-Congo haemorrhagic fever patient prevalence of Crimean-Congo haemorrhagic fever in an endemic region in Turkey. Epidemiol Infect 2014; (CCHF) in risk groups in Tokat Province of Tur- 142: 239-45

key. Afr J Microbiol Res 2010; 4: 214-7. of ribavirin is beneficial in Crimean-Congo hemorrhag- fever: systematic review and meta-analysis. BMC Inic fever. Vector Borne Zoonotic Dis 2014; 14 :300-2.

30. Yagci-Caglayik D, Korukluoglu G, Uyar Y. Sero- 36. Salehi M. Evaluation of the efficacy of ribavirin prevalence and risk factors of Crimean-Congo hem- therapy on survival of Crimean- Congo hemorrhagic orrhagic fever in selected seven provinces in Turkey. fever patients: a case-control study. Jpn J Infect Dis 2009; 62:11-15. 